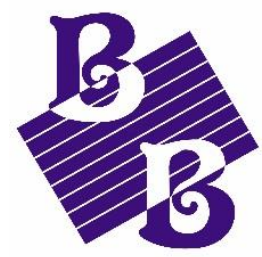

BioBacta

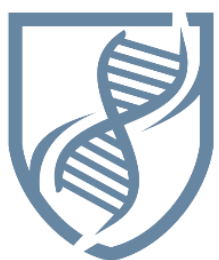

Journal of Bioscience and Applied Research

www.jbaar.org

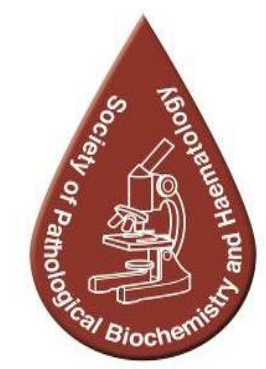

\title{
The effectiveness of nano-chlorophyll in breast cancer -targeted therapy
}

\author{
Jubran M. Abdulrahman ${ }^{1,2}$, Gihan Hosny ${ }^{2 *}$ \\ Samir A. Abd El-kaream ${ }^{3}$ \\ ${ }^{1}$ Center of Research and Educational Studies, Ministry of Education, Baghdad, Iraq. \\ ${ }^{2}$ Institute of Graduate Studies \& Research, Alexandria University, Egypt. \\ ${ }^{3}$ Medical Research Institute, Alexandria University, Egypt. \\ * To whom correspondence should be addressed: \\ Email: gihan1hosny@gmail.com;
}

Tel: (+203) 4295007; Fax: (+203) 4285792.

\section{Abstract:}

DOI: 10.21608/jbaar.2018.155993

Background: Sono-photodynamic therapy (SPDT) is a safe, non-toxic and non-invasive way of destroying cancer cells, as well as enhancing immune protective function. The present work aims to cure Ehrlich ascites carcinoma tumor implanted in a group of mice using a sonophotodynamic modality in combination with nano-chlorophyll (nano-Chl) as a sonophoto sensitizer. Methods: A total of 130 male Swiss albino mice age 60-65 days, weighing 20 $\pm 2.0 \mathrm{~g}$, were used in this experiment. Two sources of energy were used; namely infrared laser (IR) and Ultrasound for $3 \mathrm{~min}$. When the tumor had grown to about $10 \mathrm{~mm}$ in diameter at day 10 after inoculation, the treatment study was started. Six experimental groups were investigated. The dimensions of the tumor, tumor volume, tumor mass inhibition ratio, and tumor volume growth ratio were calculated. Results: The results revealed that the effect of exposing the tumor to IR laser alone or in the presence of the nano- Chl resulted in decreasing the tumor volume, tumor growth rate, and inhibition ratio. A similar result was observed in the case of using ultrasound exposure only or in the presence of the nano-Chl. Combined treatment of IR laser at $7000 \mathrm{~Hz}$ and pulsed ultrasound wave in the presence of nano-Chl was more effective than either IR laser or ultrasound alone. Conclusion: It can be suggested that folic acid-conjugated nano-Chl sono-photosensitizers (FA-NGO-Chl) could be used as a novel nanomaterial with great potential as an effective drug delivery system in targeting sono-photodynamic-therapy (SPDT).

Keywords: Sono-dynamic therapy (SDT), photodynamic therapy (PDT), Sono-photodynamic therapy (SPDT), nano-chlorophyll, Cancer treatment. 


\section{Introduction}

The most common cause of primary tumors is the genetic mutation of one or more cells, resulting in uncontrolled proliferation. The mutated cells have a proliferative characteristic over neighboring healthy cells and can form a growing mass ${ }^{(1)}$. If the mutated cells remain contained within a single cluster, with a well-defined boundary separating them from neighboring normal cells, the tumor is said to be benign, and surgical removal will often provide a complete cure. However, if the tumor cells are intermixed with normal cells and attempt to invade the surrounding tissue, the tumor is described as malignant ${ }^{(2)}$. The departure of mutated cells from the primary site represents the transition from in situ to invasive growth and is a key event in cancer progression. Subsequent entry of tumor cells into the bloodstream or lymphatic system allows access to remote parts of the body and may lead to the formation of secondary tumors (metastases), making treatment very difficult ${ }^{(3)}$. Tumor cells typically form a continuous growing cluster, which is reliant on passive diffusion for the supply of oxygen and nutrients and the removal of waste products ${ }^{(4)}$. The tumor's need for nutrients grows in proportion to its volume, but its ability to absorb diffusing substances from the surrounding tissue is proportional to its surface area. This imposes a maximum size to which the tumor can grow before it experiences nutrient deficiency. Angiogenesis provides the crucial link between the avascular and vascular states and, as such, is a key event for sustained tumor growth and cancer progression. This has raised hope of finding a cancer therapy based on anti-angiogenesis, keeping the tumor in the avascular state, in which it is usually harmless ${ }^{(5)}$.

Photodynamic therapy (PDT) is a treatment modality that uses special drugs, known as photosensitizing agents, along with light to kill cancer cells. The drugs only work after they have been activated or "turned on" by certain kinds of light. PDT may also be called photo-radiation therapy, phototherapy, or photo-chemotherapy ${ }^{(6)}$. PDT involves two steps: First, a light-sensitive drug is given. For skin cancers, it may be a cream. For internal cancers, it may be an injection into a vein, or rarely a drink. A few hours to a few days has waited before the next step. This allows time for the drug to concentrate on the cancer cells. Next, a special light (usually a laser) is shown onto cancer. The light activates the drug to treat the tumor. If the cancer is internal, an ultrasound or scan may be used to guide the light source to the tumor ${ }^{(7)}$.

Sonodynamic Therapy (SDT), is a low-intensity ultrasound that is used in clinical diagnoses, such as abdomen echo inspection, is a non-invasive treatment, and penetrates deeper into the body than light. Recently, sonodynamic therapy (SDT), ${ }^{(8)}$ which uses low-intensity ultrasound together with a sonosensitizer, has been developed for cancer therapy in applying such properties of ultrasound. So far, most sonosensitizers that have been developed are sensitive to light as well as ultrasound, implying that the shortcomings of photosensitizers used during photodynamic therapy, such as skin sensitivity, still need to be overcome in SDT. Some exceptions were, however, reported in some studies in which sensitizers were activated mainly by ultrasound but not by light. Furthermore, in vivo studies have demonstrated that SDT with a sonosensitizer has a great potential as a non-invasive and repeatable treatment for cancer therapy ${ }^{(9)}$.

Sonophotodynamic therapy (SPDT) is a safe, nontoxic and non-invasive way of destroying cancer cells, as well as enhancing immune protective function. This treatment uses the light of a particular wavelength and sound of a particular frequency to activate a light- and sonosensitive material which attaches selectively to tumor cells, causing their breakdown ${ }^{(10)}$. Both SDT and PDT have been used for years as separate processes ${ }^{(11)}$. PDT on its own is used for more superficial cancer types such as prostate, breast, and skin cancer, but when combined with SDT, it is efficient for deep-seated tumors such as bowel and ovarian cancer, as well as metastatic cancer, in particular when spread to bone, lung and liver tissues ${ }^{(12)}$. The vast majority of patients with advanced cancer treated with sono-PDT live longer than predicted and in $75 \%$ of cases, there is significant tumor cell destruction ${ }^{(13)}$. Sono-PDT is available as an effective treatment for cancer. At the beginning of the treatment, patients consume or are intravenously 
given a chlorophyll-based light-sensitive compound that binds selectively to tumor cells. It stays absorbed by cancer cells but is quickly released by healthy cells. The active agent is absorbed into the body 48$72 \mathrm{~h}$ before treatment and intravenous ozone is administered just before the treatment to enhance its effects; ozone, which is a super-oxygen compound, inhibits cancer cell growth, as cancer cells are more active under low oxygen thresholds ${ }^{(14-16)}$.

Sensitizers are the key factors for PDT and SDT. The synergistic effects of sensitizer and lowpower ultrasound have been examined in many in vitro studies and to a lesser extent in vivo models ${ }^{(17)}$. Sensitizer distribution and uptake in cells are potentially important for the therapeutic effect, because of the very short lifetime and very short diffusion distance of some radical products derived from the sensitizer produced during the procedure ${ }^{(18)}$. Different sensitizers may have different mechanisms of action. Healthy cells have an aerobic mechanism. Nanoparticles are particles between 1 and 100 nanometers in size. In nanotechnology, a particle is defined as a small object that behaves as a whole unit concerning its transport and properties.

The objectives of this study were to study the effectiveness of nanographene oxide chlorophyll in breast cancer-targeted therapy and to perform a cancer-targeted sono-photodynamic therapy utilizing the prepared nanomaterial.

\section{MATERIALS AND METHODS}

Synthesis of nano-material. In the present work nanographene oxide (NGO) was prepared using nature graphite powders as the raw materials by a modified Hummers method ${ }^{(18)}$. Also, conjugation of folic acid with graphene oxide (FA-NGO) was used. Folic acid (FA) molecules were conjugated to the NGO as described by Jönsson et al. ${ }^{(19)}$ Chlorophyll (CHL) was loaded by FA-NGO (FA-GO-CHL). Chlorophyll was used as sonophotosensitizer; chemically active by absorption of light and/or ultrasound. Chlorophyll was purchased from Molbase Chemicals Co. China. The sonophotosensitizer was obtained as a powder with a green color store in a dark Bottle at $-20^{\circ} \mathrm{C}$ temperature and with Purity: $99.9 \%$ by HPLC analysis C34H36N4O6 and MW: 596.67300 $\mathrm{g} / \mathrm{mol}$. CHL was dissolved in a sterilized buffer solution with $\mathrm{PH}=7.4$ and mixed with $\mathrm{FA}-\mathrm{NGO}$ aqueous suspension $(0.5 \mathrm{mg} / \mathrm{mL})$ at room temperature for $24 \mathrm{~h}$. Then the whole system was dialyzed against DD water for $24 \mathrm{~h}$. The standard curve was established in one range of drug concentration. UVVis measurements of FA-NGO- CHL in the mixed solvent $(0.1 \mathrm{~mL}$ ethanol $+2.9 \mathrm{~mL} \mathrm{DD})$ were carried out. The loading efficiency of Chlorophyll was calculated according to UV absorbance at $663 \mathrm{~nm}$. Every experiment was repeated three times. FANGO- CHL administered to tumor-bearing mice intraperitoneal (IP) injection for 15 days 18-20 hours before exposure to either photo and/or sonodynamic treatment modality.

Animal treatment. A total of 130 male Swiss albino mice age 60- 65 days, weighing $20 \pm 2.0 \mathrm{~g}$, were purchased from the National Cancer Institute, Cairo University, were induced Ehrlich Tumor Cells. Ehrlich ascites carcinoma tumor cells, $2 \times 10^{6}$ mammary in origin, diluted approximately (1-9) in $0.9 \%$ saline were inoculated subcutaneously on the left side of mice. The animals were housed in plastic cages and were kept under natural light with diet and water available. When the tumor had grown to about $10 \mathrm{~mm}$ in diameter at day 10 after inoculation, the treatment study was started. The use of experimental animals in the study protocol was carried out following the ethical guidelines of the Medical Research Institute, Alexandria University (Guiding Principles for Biomedical Research Involving Animals, 2011).

The experimental animal treatment groups were as follows:

\section{Group I: (30 mice)}

a) $\mathbf{1 0}$ mice: Control without tumor.

b) 10 mice: Tumor-bearing mice without treatment.

c) 10 mice: Tumor-bearing mice treated with (FA-NGO-CHL) only.

\section{Group II: (20 mice, laser-irradiated group)}

a) 10 mice: we're exposed to Infra-Red Laser $(4000 \mathrm{~Hz})$ for 3 minutes. 
b) $\mathbf{1 0}$ mice: we're exposed to Infra-Red Laser $(7000 \mathrm{~Hz})$ for 3 minutes.

\section{Group III: (20 mice, ultrasound group)}

a) 10 mice: were exposed to pulsed ultrasound for 3 minutes.

b) 10 mice: were exposed to continuous ultrasound for 3 minutes.

\section{Group IV: (20 mice, (FA-NGO- CHL), laser group)}

The mice of this group were injected intraperitoneally (IP) with (FA-NGO- CHL), then the tumor site will be irradiated to laser light at the same conditions of group II.

\section{Group V: (20 mice, (FA-NGO- CHL), ultrasound group)}

The mice of this group were injected (IP) with (FA-NGO- CHL), then were divided into 2 sub-groups. The tumor site was irradiated to ultrasound at the same conditions as group III.

\section{Group VI: (20 mice, combined treatment groups)}

a) $\mathbf{1 0}$ mice: The tumor site was irradiated to laser light $(7000 \mathrm{~Hz})$ for 3 minutes, followed by pulsed ultrasound for 3 minutes.

b) 10 mice: Injected (IP) with (FA-NGO- CHL). The tumor sites were irradiated to laser light (7000 Hz) for $3 \mathrm{~min}$, followed by pulsed ultrasound for 3 minutes.

For the laser exposure, the mice were anesthetized with diethyl ether. The hair over the tumors was shaved off. The mice were fixed on a board with the tumor upwards. The probe was placed nearly on the tumor, which was irradiated with laser for three minutes at the different conditions as mentioned before. After PDT, animals were maintained in the dark to avoid skin irritation.
Exposure of mice tumor to the laser beam was carried out using an Infrared diode laser, model LAS 50- Hi-Tech Fysiomed, Germany operated at a wavelength of $904 \mathrm{~nm}$ and peak power of $50 \mathrm{~W}$ at a frequency up to $7000 \mathrm{~Hz}$. The unit has a large menu of preset pathologies. For each pathology, all parameters are preset and stored in the memory, but can always be modified by a therapist.

For the ultrasound exposure, the mice were anesthetized with diethyl ether. The hair over the tumors was shaved off. The mice were fixed on a board with the tumor upwards. After added gel locally the probe was placed nearly on the tumor, which was irradiated with ultrasound for three minutes at the different conditions as mentioned before. Exposure of Ehrlich Tumor was carried out using an ultrasonic therapy instrument (Model CSl Shanghai, No. 822 Factory. China). This instrument uses an electronic tube to generate an electric oscillation with a frequency of $0.8 \mathrm{MHz}$ and power output which is converted to ultrasonic mechanical energy using an ultrasonic transducer (calcium zirconate -titanate). The mechanical ultrasonic energy has a beam power density that can be adjusted from 0.5 to $3 \mathrm{~W} / \mathrm{cm}^{2}$. Sonocation time can be adjusted up to 30 minutes, while the set-time is over, the power supply is cut off automatically and intermittent alarming sound may be given. This instrument operates at both continuous wave mode with output power from $0.5-3 \mathrm{~W} / \mathrm{cm}^{2}$ adjustable in 11 steps and pulsed mode (pulse frequency $1000 \mathrm{~Hz}$, duty ratio $1 / 3$, and average power density from $0.15-1$ $\mathrm{W} / \mathrm{cm}^{2}$ ).

Tumor Growth Assay. During a treatment session, tumor growth was examined regularly every day. The length and width of tumors were measured with a slide caliper and tumor volume was calculated by the use of the following equation. ${ }^{(20)}$

$$
\text { Tumor volume }(\mathrm{mm} 3)=\frac{22}{7} \times \frac{4}{3} \times \frac{\text { Length }}{2} \times\left(\frac{\text { Wedth }}{2}\right)^{2}
$$




\section{Evaluation of Sonophotosensitizer and SPDT}

Two weeks after the treatment, the mice were sacrificed and the tumors were dissected out, weighed (in grams), their volumes were measured using cylindrical measuring flux. The tumor mass inhibition ratio and tumor volume growth ratio were calculated as follows ${ }^{(20)}$.

\section{TumorMassInhibitionRatio $(T M I R)=$}

$$
\left(1-\frac{\text { Averagetumorweightofthetreatedgroup }}{\text { Averagetumorweightof thecontrol }}\right) \times 100
$$

\section{Statistical analysis}

Analysis of numeric data was performed using one-way ANOVA; it is a parametric statistical test that is used to compare the means for certain data of more than two independent groups which follow a normal distribution. The given graphs were constructed using Microsoft Excel software. All statistical analysis was done using two-tailed tests and an alpha error of 0.05 . A P-value less than or equal to 0.05 was considered to be statistically significant.

\section{RESULTS}

The underlying work was conducted aiming at curing Ehrlich ascites carcinoma tumor implanted in a group of mice, using a sonophotodynamic modality in combination with nano-Chl as a sonophoto sensitizer drug. Two sources of energy were used; namely infrared laser at two frequency levels (4000 and $7000 \mathrm{~Hz}$ ) with power density $16.8 \mathrm{~mW} / \mathrm{cm}^{2}$ and Ultrasound (pulsed and continuous wave mode) at power density $3 \mathrm{~W} / \mathrm{cm}^{2}$ for $3 \mathrm{~min}$. Effects of Treatment Modalities on Tumor Volume: The relationships between tumor volumes and treatment period for various treatment modalities (treated with IRL and ultrasound (pulsed or continuous wave) in the presence or absence of photosensitizer are presented in Figures 1 to 5. Tumor volumes were normalized to volumes before starting the treatment. Treatment with photosensitizer has little or no effect on tumor volume. Up to one week, all treatment modulates have little or no effect on the tumor volume. After one week, treatment with IRL and ultrasound (pulsed or continuous wave) in the presence or absence of photosensitizer, becomes more effective. The presence of a photosensitizer increases the effect of both IRL and ultrasound. Results obtained indicated that a pulsed ultrasonic wave is more effective than a continuous ultrasonic wave in the presence of a photosensitizer. Pulsed wave ultrasound at $3 \mathrm{~W} / \mathrm{cm}^{2}$ was selected to combine with IRL at 7000 $\mathrm{Hz}$. This combined treatment modality is more effective on tumor cells than using an infrared laser (IRL) or ultrasound alone. 


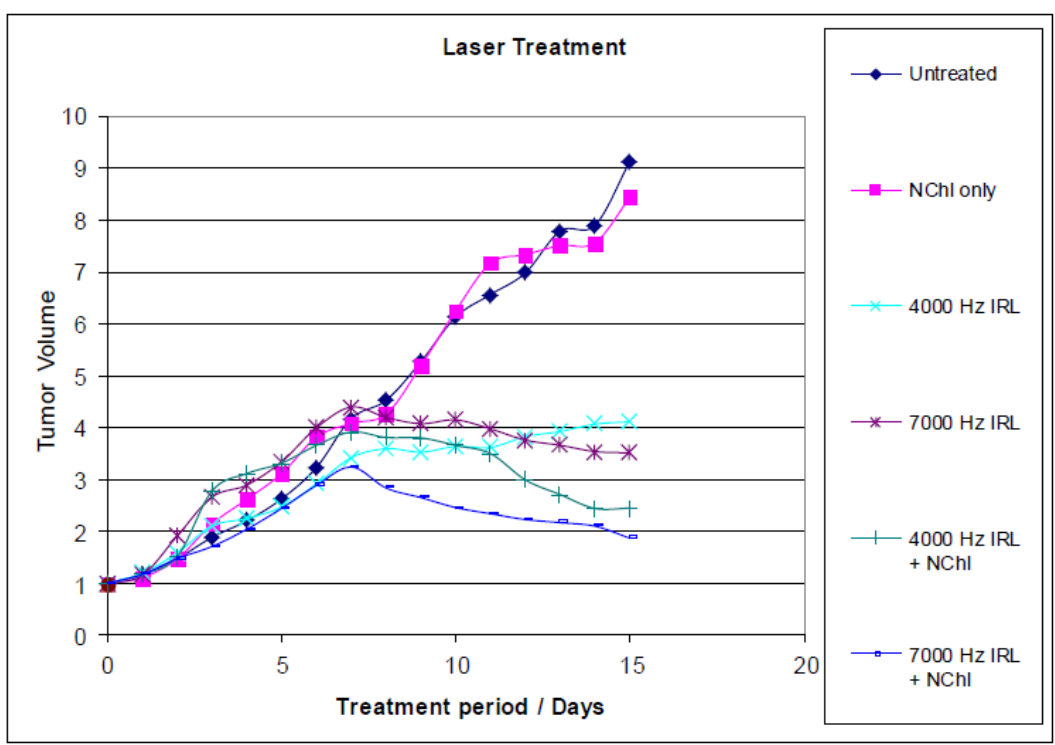

Figure 1. The Effect of IRL treatment period at different frequencies on the tumor volume $\left(\mathrm{mm}^{3}\right)$.

Figures 2 to 4 represent the average tumor volume, at the end of the IRL treatment, US treatment (pulsed and continuous) respectively. The treatment with IRL alone decreased the tumor volume by increasing the IRL frequency. Also, at the end of the ultrasound treatment, the average tumor volume decreased more profoundly in the group subjected to pulsed than the contiguous US.

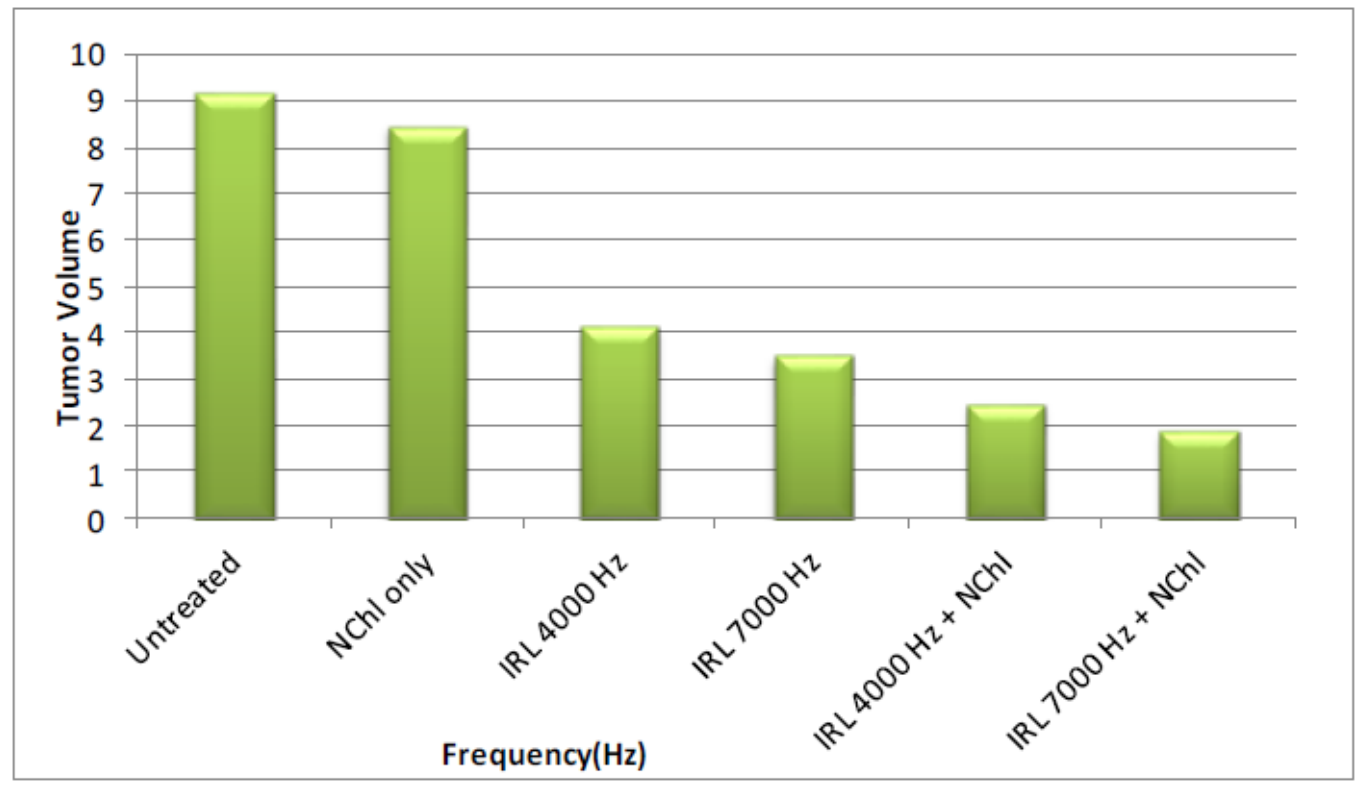

Figure 2. The average tumor volume $\left(\mathrm{mm}^{3}\right)$ at day 15 of treated and untreated IRL groups. 


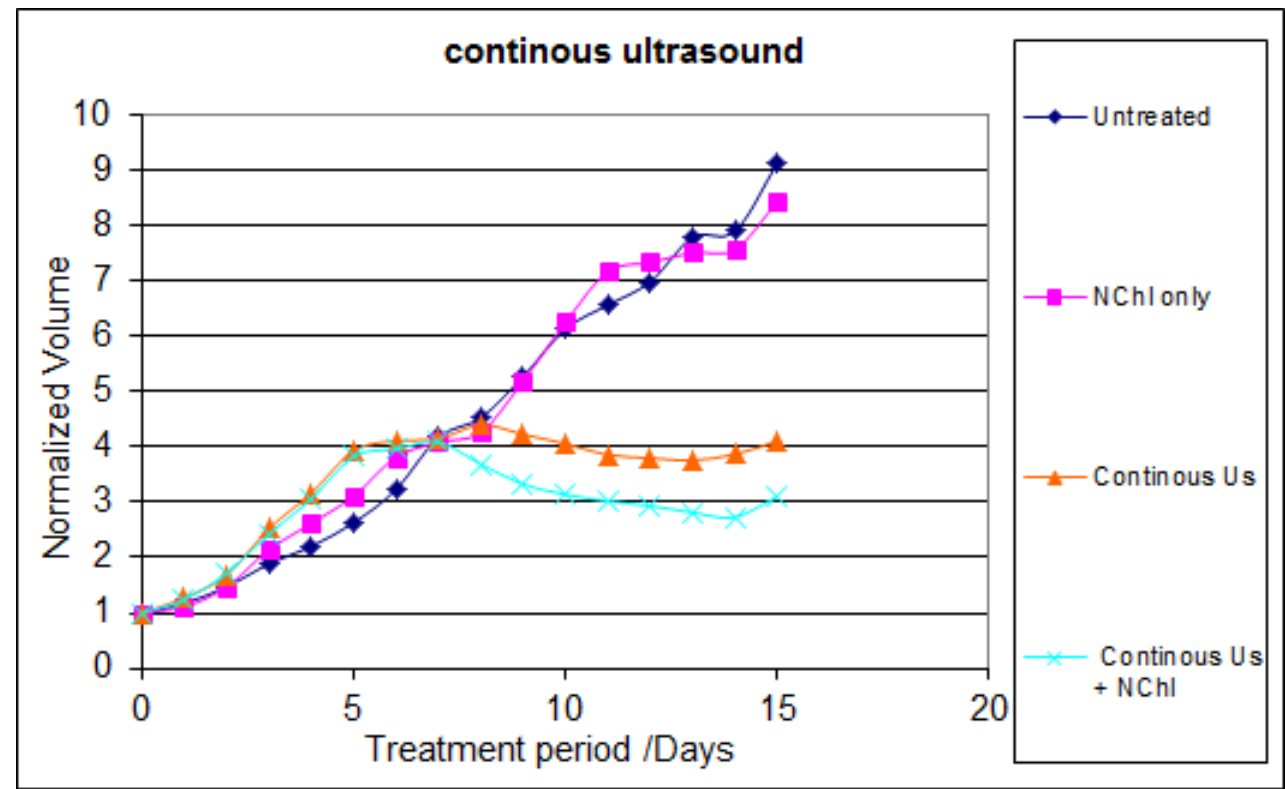

Figure 3. The Effect of continuous-wave ultrasound $\left(3 \mathrm{~W} / \mathrm{cm}^{2}, 0.8 \mathrm{MHz}, 1 \mathrm{~min}\right)$ on tumor volume $\left(\mathrm{mm}^{3}\right)$ with and without photosensitizer.

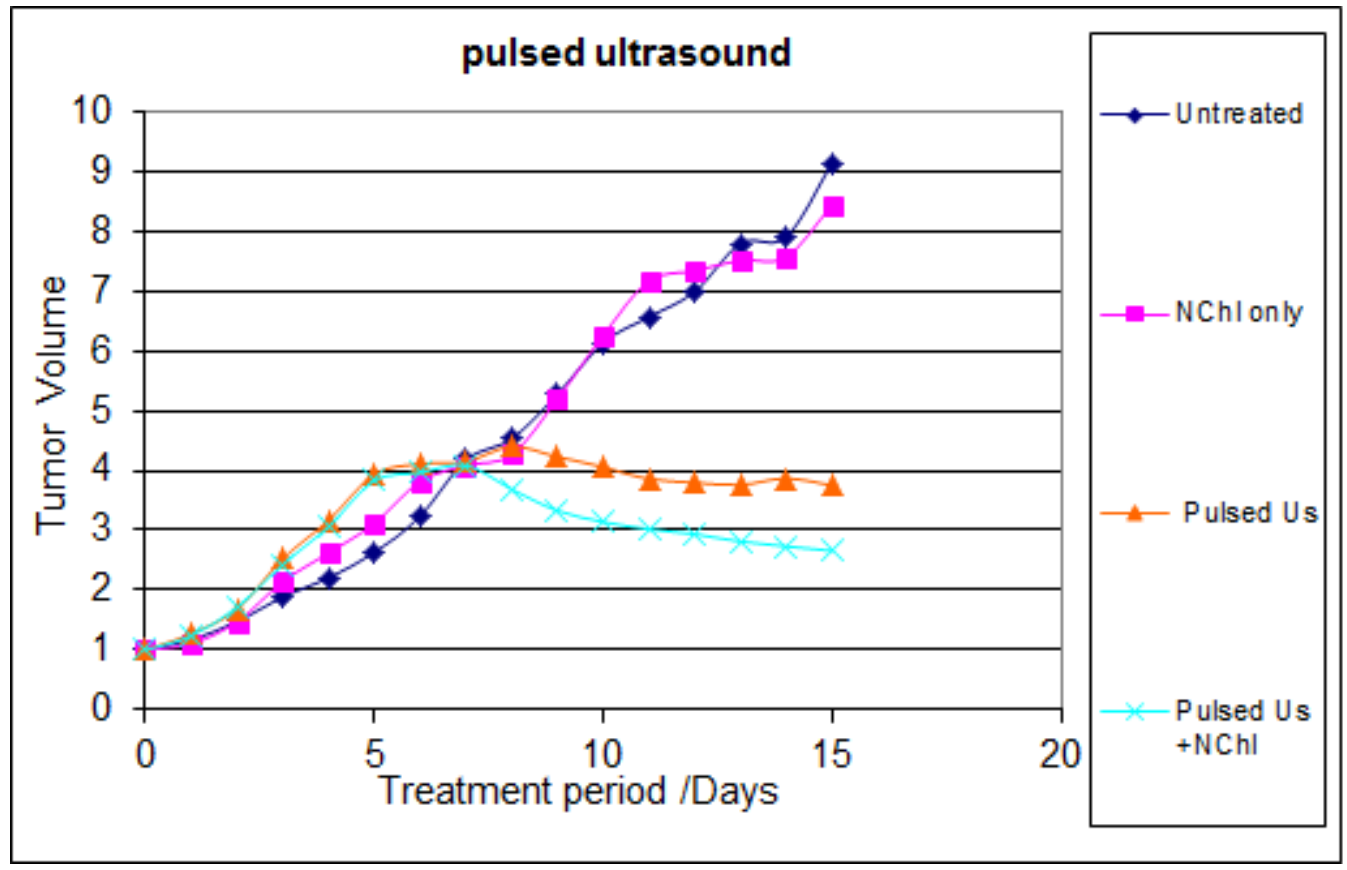

Figure 4. The Effect of pulsed wave ultrasound $\left(3 \mathrm{~W} / \mathrm{cm}^{2}, 0.8 \mathrm{MHz}, 1 \mathrm{~min}\right)$ on tumor volume $\left(\mathrm{mm}^{3}\right)$ with and without photosensitizer. 


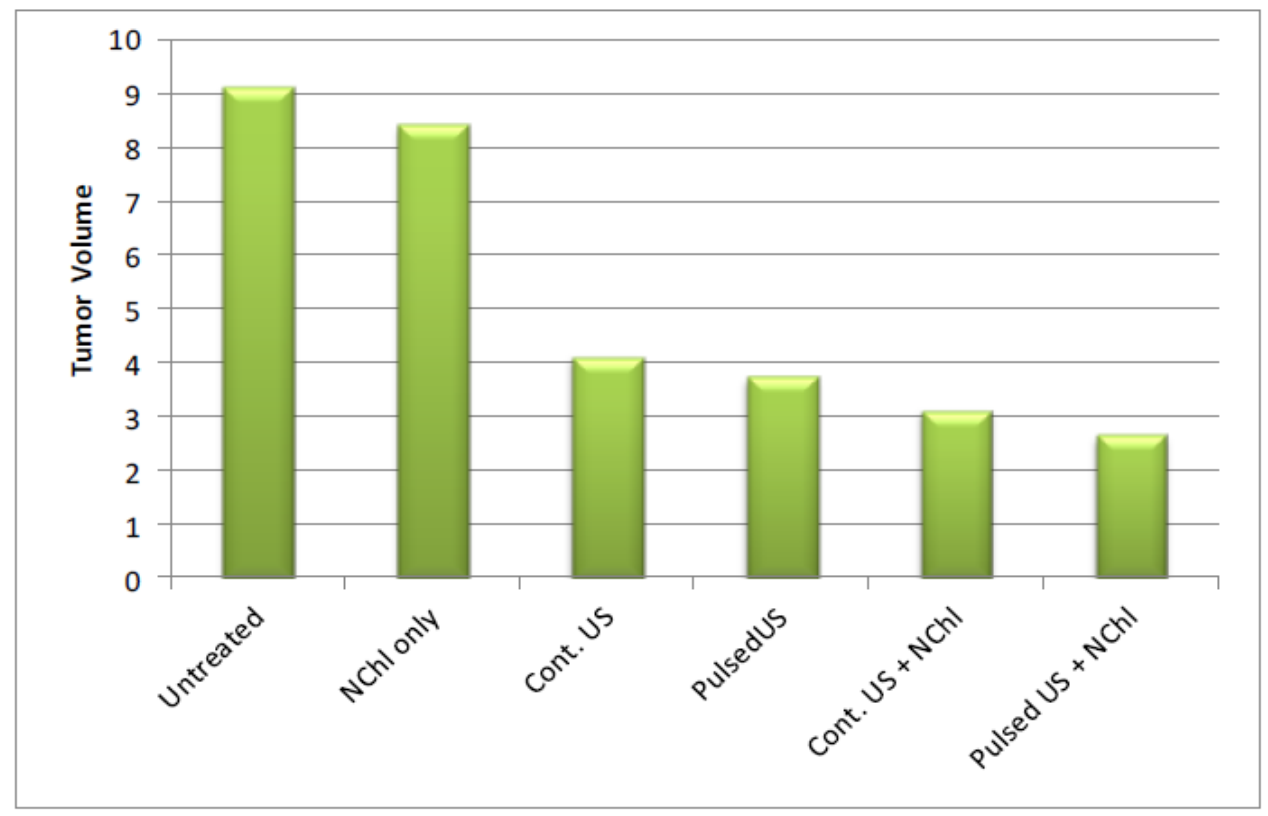

Figure 5. The average tumor volume $\left(\mathrm{mm}^{3}\right)$ at day 15 for EAC treated group and groups exposed to ultrasound (cont. and pulsed), and NChl.

Figures 6 and 7 describe the effect of combined treatment with both IRL and ultrasound waves. It can be concluded that this combination is more effective (the last bar to the right) than the treatment with IRL or ultrasound alone.

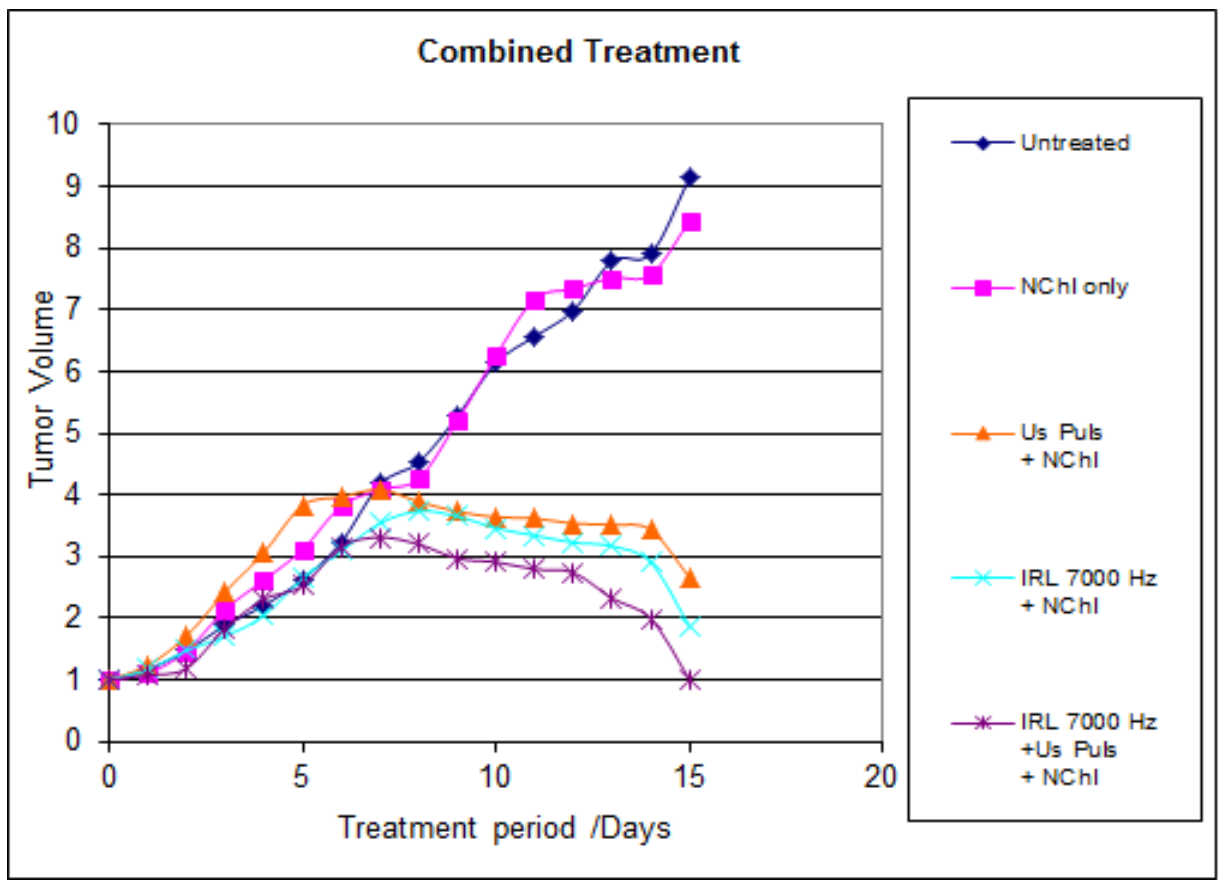

Figure 6. The Effect on tumor volume $\left(\mathrm{mm}^{3}\right)$ of combined exposure (IRL and ultrasound) in the presence of a photosensitizer. 


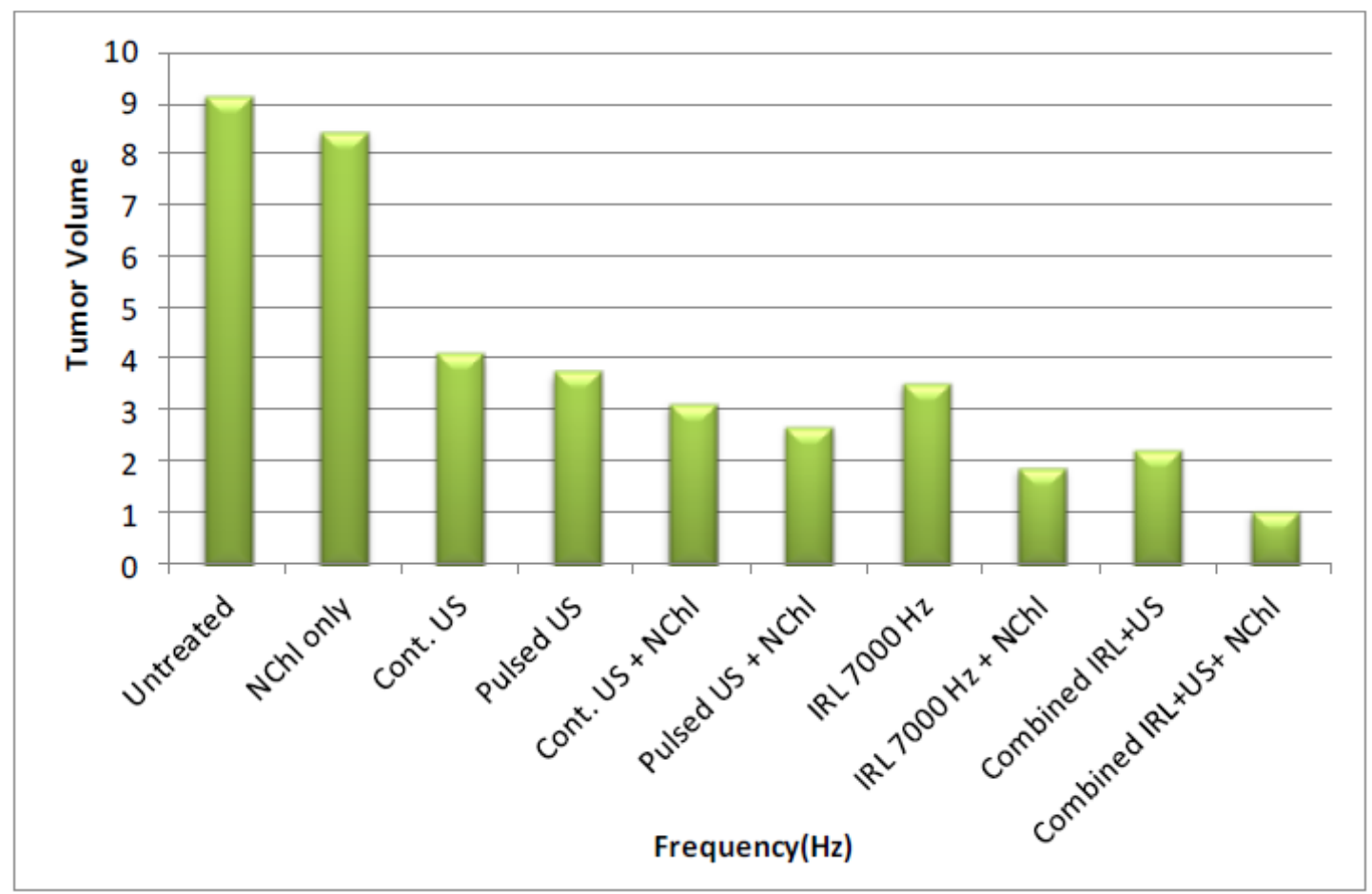

Figure 7. The average tumor volume $\left(\mathrm{mm}^{3}\right)$ at day 15 of treatment for EAC treated group and groups exposed to ultrasound wave and /or IRL.

Tumor Volume Growth Ratio (TVGR): Table 1 shows the tumor volume growth rate for only EAC and treated groups. NChl photosensitizer alone had no inhibitory effect on tumor growth rate. IRL alone at $7000 \mathrm{~Hz}$ had a lower growth rate than that of ultrasound wave (pulsed or continuous). IRL operated at $4000 \mathrm{~Hz}$ and $7000 \mathrm{~Hz}$ combined with photosensitizer showed synergistic antitumor effect than ultrasound with a photosensitizer. A combination of IRL at $7000 \mathrm{~Hz}$, pulsed wave ultrasound at 3 $\mathrm{W} / \mathrm{cm}^{2}$, and photosensitizer showed high suppression of tumor growth rate. The tumor growth rate of the combined treated group is nearly nine times lower than that of only the EAC group. ANOVA test revealed statistically significant differences between groups at $\mathrm{p} \leq 0.05$. 
Table 1. Tumor volume and tumor volume growth ratio in the different studied groups at the end of 15 days of treatment.

\begin{tabular}{|c|c|c|c|}
\hline Groups & Tumor volume $\left(\mathrm{mm}^{3}\right)$ & $\begin{array}{l}\text { Inhibition ratio } \\
(\%)\end{array}$ & $\begin{array}{l}\mathbf{F} \\
(\mathbf{p})\end{array}$ \\
\hline Only with EAC & $9.13 \pm 1.1$ & 100 & \multirow{18}{*}{$\begin{array}{l}53.952 \\
(\mathbf{p}<0.001 *)\end{array}$} \\
\hline NChl & $8.44 \pm 1.3$ & 92.442 & \\
\hline \multicolumn{3}{|l|}{ IRL } & \\
\hline $4000 \mathrm{~Hz}$ & $4.11 \pm 0.41$ & 45.016 & \\
\hline $7000 \mathrm{~Hz}$ & $3.51 \pm 0.43$ & 38.444 & \\
\hline \multicolumn{3}{|l|}{ IRL + NChl } & \\
\hline $4000 \mathrm{~Hz}$ & $2.45 \pm 0.40$ & 26.835 & \\
\hline $7000 \mathrm{~Hz}$ & $1.87 \pm 0.31$ & 20.482 & \\
\hline \multicolumn{3}{|l|}{ Ultrasound } & \\
\hline Continuous US & $4.11 \pm 0.42$ & 45.016 & \\
\hline Pulsed US & $3.75 \pm 0.48$ & 41.073 & \\
\hline \multicolumn{3}{|l|}{ Ultrasound + NChl } & \\
\hline Continuous US & $3.11 \pm 0.31$ & 34.064 & \\
\hline Pulsed US & $2.66 \pm 0.30$ & 29.135 & \\
\hline \multicolumn{3}{|l|}{ IRL + Ultrasound } & \\
\hline $7000 \mathrm{~Hz}+$ Pulsed US & $2.2 \pm 0.11$ & 24.096 & \\
\hline \multicolumn{3}{|l|}{ IRL + Ultrasound + NChl } & \\
\hline $7000 \mathrm{~Hz}+$ Pulsed US + NChl & $1.01 \pm 0.03$ & 11.062 & \\
\hline
\end{tabular}

F: F value for ANOVA test

*: Statistically significant

Tumor Mass Inhibition Ratio (TMIR): Table 2 illustrates the effect of different treatment modalities at the end of the treatment period of 15 days. It is clear from this table that the tumor weight after IRL or ultrasound in the presence of photosensitizer was reduced compared with that after IRL or ultrasound alone. The maximum inhibition ratio percentage was noticed in the treated group with IRL and ultrasound in the presence of a photosensitizer. ANOVA test revealed statistically significant differences between groups at $\mathrm{p} \leq 0.05$. 
Table 2. Tumor weights and tumor mass inhibition ratio in the different studied groups at the end of 15 days of treatment.

\begin{tabular}{|c|c|c|c|}
\hline Groups & Tumor weight (g) & Inhibition ratio $(\%)$ & $\begin{array}{l}\mathbf{F} \\
\text { (p) }\end{array}$ \\
\hline Only with EAC & $6.32 \pm 1.21$ & 00.000 & \multirow{18}{*}{$\begin{array}{l}30.294 \\
(\mathrm{p}<0.001 *)\end{array}$} \\
\hline NChl & $6.16 \pm 1.30$ & 2.532 & \\
\hline \multicolumn{3}{|l|}{ IRL } & \\
\hline $4000 \mathrm{~Hz}$ & $1.77 \pm 0.43$ & 71.993 & \\
\hline $7000 \mathrm{~Hz}$ & $1.65 \pm 0.44$ & 73.892 & \\
\hline \multicolumn{3}{|l|}{ IRL + NChl } & \\
\hline $4000 \mathrm{~Hz}$ & $0.88 \pm 0.41$ & 86.075 & \\
\hline $7000 \mathrm{~Hz}$ & $0.67 \pm 0.31$ & 89.399 & \\
\hline \multicolumn{3}{|l|}{ Ultrasound } & \\
\hline Continuous US & $1.45 \pm 0.69$ & 77.056 & \\
\hline Pulsed US & $1.18 \pm 0.71$ & 81.329 & \\
\hline \multicolumn{3}{|l|}{ Ultrasound + NChl } & \\
\hline Continuous US & $1.02 \pm 0.32$ & 83.860 & \\
\hline Pulsed US & $0.97 \pm 0.33$ & 84.652 & \\
\hline \multicolumn{3}{|l|}{ IRL + Ultrasound } & \\
\hline $7000 \mathrm{~Hz}+$ Pulsed US & $0.64 \pm 0.14$ & 89.873 & \\
\hline \multicolumn{3}{|l|}{ IRL + Ultrasound + NChI } & \\
\hline $7000 \mathrm{~Hz}+$ Pulsed US + NChl & $0.45 \pm 0.01$ & 92.870 & \\
\hline
\end{tabular}

F: F value for ANOVA test

*: Statistically significant

\section{DISCUSSION}

The underlying work was conducted aiming at obtaining more positive results on using the SPDT in combination with sono-photosensitizer loaded on graphene oxide nanoparticles as an up-to-date treating cancer modality to cure Ehrlich ascites carcinoma, EAC. Results revealed that Nano-Chl is a potential photosensitizer and sonosensitizer for photodynamic or sonodynamic treatment of Ehrlich ascites tumor tissue. Nano-Chl can play important roles in inhibiting tumor growth and even inducing cell death, which might be attributed to the Photo or sonochemical activation mechanism. Infra-red laser in combination with ultrasound in the presence of nano-Chl has a potential antitumor effect. Sonocation followed by light photon irradiation proves its excellent efficiency as an anticancer therapy. The results suggest that folic acid-conjugated nanographene oxide loaded with sono-photosensitizers (FA-NGO-SPSs) could be used as a novel nanomaterial with great potential as an effective drug delivery system in targeting sono-photodynamictherapy (SPDT). Cancer is a class of diseases or disorders characterized by an uncontrolled division of cells and the ability of these cells to invade other tissues, either by direct growth into adjacent tissue 
through invasion or by implantation into distant sites by metastasis. This unregulated growth is caused by damage to DNA, resulting in mutations to genes that encode for proteins controlling cell division. Many mutation events may be required to transform a normal cell into a malignant cell. These mutations can be caused by chemicals or physical agents called carcinogens, or by certain viruses that can insert their DNA into the human genome. Mutations occur spontaneously or are passed down generations as a result of germline mutations ${ }^{(21)}$. Cancer can be treated by surgery, chemotherapy, radiation therapy, immunotherapy, and electro-chemo-therapy, or other modalities. The choice of therapy treatment method depends upon the location and grade of the tumor and the stage of the disease, as well as the general state of the case. Complete removal of cancer without damage to the rest of other organs or tissues is the goal of treatment. Sometimes this can be accomplished by surgery, but the propensity of cancers to invade adjacent tissue or to spread to distant sites by microscopic metastasis often limits its effectiveness. The effectiveness of chemotherapy is often limited by toxicity to other tissues in the body. Radiation can also cause damage to normal tissue, which is also a general drawback of this cancer treatment therapy ${ }^{(22)}$.

Photodynamic therapy (PDT), involves the administration of photosensitizing drugs and subsequent exposure of the tissue to light. This modality has emerged as a novel clinical approach for the treatment of various tumors and some other nonmalignant conditions ${ }^{(23,24)}$. Due to the selectivity of drug uptake ${ }^{(25)}$ and the control of light delivery, PDT has the potential of inducing effective cytotoxicity in malignant tissue and limited damage to the surrounding healthy tissues. However, despite the progress in the development of this method, relapses sometimes emerge after therapy ${ }^{(26)}$, and hence, a combination of PDT with other methods used for cancer treatment seems to be a promising trend. One of these methods, though studied by the present time, is sonodynamic therapy (SDT). SDT was derived from photodynamic therapy (PDT) in $1989^{(27)}$. PDT, which generates cytotoxic singlet oxygen via activating photosensitizer at a specific wavelength, is a minimally invasive procedure with increasing promise in the treatment of malignant and nonmalignant diseases. ${ }^{(28,29)}$ Since ultrasound can penetrate tissue more deeply than light, SDT is easier to interact with the cells buried in tissue deeply than PDT. ${ }^{(30)}$ Reactive oxygen species (ROS), especially singlet oxygen $\left({ }^{1} \mathrm{O}_{2}\right)$, is the key effecter for both therapeutic methods to cause oxidative damage of cellular components ${ }^{(31,32)}$. In the present work, a combined treatment of photosensitizer, IR laser photodynamic therapy, and sonodynamic therapy were employed the purpose was to investigate whether IR laser, ultrasound, and nano-Chl as photosensitizer alone or combined, could be safely administered and provide an increased local tumor cytotoxic response.

\section{Conclusion}

It can be concluded that the present study opened new trends for cancer treatment therapy that needs to be further verified. The study gave profound results involving the use of sono-photodynamic modality employing exposure to infra-red laser and ultrasound with (pulsed and continuous) in combination with nano-Chl as a sono-photosensitizer for treating Ehrlich Ascites Carcinoma tumor implanted to mice as an experimental animal. The possible application of nanocarrier-sonophotodynamic therapy as in vivo anti-malignancy can open a new line of research for modern cancer therapy that needs to be further investigated. Nanomaterial with their great potential for effective drug delivery can permit the feasibility of targeted therapy for disease treatment that needs further research for optimizing and maximizing benefits. Conjugated nanomaterial therapy can potentially provide a very valuable application for amplifying the benefits of photodynamic therapy. A response can be improved utilizing sonodynamic targeted therapy to treat deep or multiple lesions simultaneously. Further research is required to validate this novel therapy to prove the feasibility and safety of the application.

\section{References}

1. King RJ. Natural history: the life of a cancer. In: Cancer Biology; 3rd ed. King RJ, Robins MW; eds. Longman Essex (pub.), 2006, chapter 1, pp.9-31.

2. Plank MJ, Sleeman BD. Tumour-induced Angiogenesis: a Review. J Theor Med 2003; 5: 137-53.

3. Schirrmacher V. Cancer metastasis: experimental approaches, theoretical concepts and impacts for treatment strategies. AdvCanc Res 1985; 43: 1-73. 
4. Sutherland RM. Cell and environment interactions in tumor micro-regions: the multicell spheroid model. Science 1988; 240: 177-84

5. Folkman J. Tumor angiogenesis: therapeutic implications. N Engl J Med 1971; 285: 11826.

6. Henderson B, Busch T and Snyder J. Fluence rate as a modulator of PDT mechanisms. Lasers Surg Med 2006; 30: 15-26.

7. Berg H. Photodynamic tumour therapy and cancer multistep therapy. Journal of Photochem Photobiol B: Biol 1988; 2: 404-9.

8. Shibaguchi H, Tsuru H, Kuroki M, Kuroki M. Sonodynamic cancer therapy: a non-invasive and repeatable approach using low-intensity ultrasound with a sonosensitizer. Anticancer Res 2011; 3: 2425-9.

9. Trendowski M. Using the Promise of Sonodynamic Therapy in the Clinical Setting against Disseminated Cancers. Chemother Res Prac 2015; 2015: 316015. Published online $2015 \quad$ Aug 25. DOI: $10.1155 / 2015 / 316015$.

10. Kennedy JC, Pottier RH. New trends in photobiology: endogenous protoporphyrin IX, a clinically useful photosensitizer for photodynamic therapy. J Photochem Photobiol B: Biol 1992; 14: 275-92.

11. Batlle AD. Porphyrins, porphyrias, cancer and photodynamic therapy - a model for carcinogenesis. J Photochem Photobiol B: Biol 1993; 20: 5-22.

12. Orenstein A, Kostenich G, Kopolovic Y, Babushkina T, Malik Z. Enhancement of ALA-PDT Damage by IR-induced Hyperthermia on a Colon Carcinoma Model. Photochem Photobiol 1999; 69: 703-7.

13. Key TJ. Fruit and vegetables and cancer risk. Br J Cancer 2011; 104: 6-11.

14. Dolmans DE, Fukumura D, Jain RK. Photodynamic therapy for cancer. Nature Rev Cancer 2003; 3: 380-7.
15. Chen B, Pogue BW, Hoopes PJ, Hasan T. Combining vascular and cellular targeting regimens enhances the efficacy of photodynamic therapy. Inter J Rad Oncol Biol Phys 2005; 61: 1216-26.

16. Berns MW, Coffey J, Wile AG. Laser photoradiation therapy of cancer: possible role of hyperthermia. Lasers Surg Med 1984; 4: 87-92.

17. Dougherty TJ. Photodynamic therapy. Photochem Photobiol 1993; 58: 895-900.

18. Hummers JrW, Offeman R. Preparation of graphitic oxide. J Am Chem Soc 1958; 80: 1339-44.

19. Johnsson B, Lofas S, Lindquist G. Immobilization of proteins to a carboxymethyldextran-modified gold surface for biospecific interaction analysis in surface plasmon resonance sensors. Anal Biochem 1991; 198: 268-77.

20. Honda H, Zhao QL, Kondo T. Effects of dissolved gases and an echo contrast agent on apoptosis induced by ultrasound and its mechanism via the mitochondria-caspase pathway. Ultras Med Biol 2002; 28: 673-82.

21. Vogelstein B, KW. "Cancer genes and pathways they control". Nat Med 2004; 10: 220-5.

22. Murphy GP, Morris LB., and Lange. "Informed decision: The complete book of cancer diagnosis, treatment, and recovery" Viking Penguin: the American Cancer Society. 1997.

23. Ackroyd R, Kelty C, Brown N, et al. The history of photodetection and photodynamic therapy. Photochem Photobiol 2001; 74: 656 - 669.

24. Detty M, Gibson S and Wagner S. Current clinical and preclinical photosensitizers for use in photodynamic therapy. J Med Chem 2004; 47: 3897-915.

25. Mugherty $\mathrm{T}$, Grindey $\mathrm{G}$, Fiel R, et al. Photoradiation therapy. II cure of animal tumors with hematoporphyrin and light. J Natl Cancer Inst 1975; 55: 115- 21. 
26. Mikhailovskaya A, Kaplan M, Brodskij R, et al.Combined Exposure to Electrochemical Lysis and Photodynamic Therapy.Bulletin of Experimental Biology and Medicine. 2009; 147: 95-8.

27. Umemura S, Yumita N, Nishifaki R, et al. Sonochemical activation of hematoporphyrin: A potential modality for cancer treatment. Proc IEEE Ultrason. Symp 1989; 9: 955-60.

28. Doughterty T, Gomer C, Henderson B, et al. Photodynamic therapy: Review. J natle Cancer Inst 1998; 90: 889-905.

29. Ortner M. Photodynamic therapy for cholangiocarcinoma. J Hepatobiliary Pancreat Surg 2001; 8: 137-39.
30. Wong T, Tracy E, Oseroff A, et al. Photodynamic therapy mediates immediate loss of cellular responsiveness to cytokines and growth factors. Cancer Res 2003; 63: 3812-18.

31. Dolmans DE, Fukumura D, Jain RK. Photodynamic therapy for cancer. Nat Rev Cancer 2003; 90: 889-905.

32. Rosenthal I. Sostaric J and Riez P. Sonodynamic therapy - A review of the synergistic effect of drugs and ultrasound. Ultrason Sonochem 2004; 11: 349-63. 